\title{
IMPACTO DE LA GLOBALIZACIÓN EN LAS PRÁCTICAS SOCIOECONÓMICAS DE LA COMUNIDAD TIKUNA DEL RESGUARDO DE MOCAGUA - AMAZONAS ${ }^{1}$ \\ Impact of Globalization on the Socioeconomic Practices of the Tikuna Community of the Resguardo De Mocagua - Amazonas
}

${ }^{1}$ Este artículo recoge algunos de los resultados de la tesis de pregrado que los autores presentaron al programa de Trabajo Social de la Facultad de Ciencias Sociales de la Universidad Colegio Mayor de Cundinamarca, en junio de 2016. Los autores agradecen a Parques Nacionales Naturales de Colombia Regional Amazonia, y al Parque Nacional Natural Amacayacu, así como a la comunidad de Mocagua quienes dieron su permiso y acompañamiento en el trabajo de campo.
David L. Guantiva Pinzón ${ }^{2}$ Juan S. Valencia Sánchez ${ }^{3}$

2Docentes, Universidad Colegio Mayor de Cundinamarca ${ }^{3}$ Docentes, Universidad Colegio Mayor de Cundinamarca

Recibido: 15 de mayo 2019 // Aceptado: 28 de junio de 2019 // Publicado: 8 de julio de 2019

\section{Resumen}

El proyecto investigativo tuvo como objetivo conocer cuáles han sido las transformaciones socioeconómicas que ha tenido la comunidad Tikuna de Mocagua desde los procesos de globalización en el Trapecio Amazónico. La investigación es de tipo cualitativo de corte etnográfico, se diseñan instrumentos para el trabajo de campo orientados al Identificar las transformaciones a lo largo del tiempo de la comunidad, con el objetivo de establecer cuál es su postura frente a estos procesos, partiendo del análisis de las categorías propuestas en el cuerpo de la investigación y aquellas emergentes durante el proceso de interacción con los sujetos. A partir de ello se encuentran transformaciones considerables en su organización política, el manejo de recursos y del dinero, así como problemáticas sociales modernas que obligan a la comunidad a adaptarse a un mundo globalizado, intentando proteger sus tradiciones y su entorno.

\section{Palabras claves}

Amazonas, globalización, práctica socioeconómica, transformación, Tikuna.

\begin{abstract}
The investigation had as main objective to find out what has been the socio-economic transformations that the Tikuna Community of Mocagua has had from the processes of globalization in the Amazon trapezium, considering that the research is qualitative and ethnographic in nature, instruments are designed for fieldwork directed at: the past, with the aim of identifying traditional elements and the community's history; the present, to identify the current situation of the community in relation to the objective of study and the future with the intention to investigate the position of the community and its proposal in the face of living situations.
\end{abstract}

\section{Key words}

Amazon, globalization, socioeconomic practice, transformation, Tikuna.

\section{Introducción}

El pueblo originario Tikuna se ha caracterizado por adaptarse a las diversas condiciones del contacto intercultural con otros grupos indígenas y no indígenas, a través de movimientos espaciales, negociaciones políticas y prácticas religiosas. La información recolectada de los estudios etnográficos llevados a cabo entre los Tikuna ha hecho énfasis en el proceso histórico de este grupo y su proceso de contacto, el cual está estrechamente relacionado con la historia del trapecio amazónico. (Soto 2010,10).

En primer lugar (Fontaine, 2006) en su estudio 
sobre El mambe frente al dinero entre los Yucuna del Amazonas, señala que:

... grupos manejan pesos colombianos principalmente en sus relaciones de intercambio con los blancos. Esta adquisición de dinero es producto de relaciones de trabajo entre los grupos indígenas y los blancos, o de comercio de mercancías, llevándolos una vez más a la evidencia de la inserción de necesidades provenientes del mercado moderno en la vida diaria de estos grupos. (p.6).

Por otro lado da Silveira, en su estudio sobre agricultores, pescadores y recolectores de una villa del amazonas, identificó que:

...la actividad agrícola de los indígenas Quatipuru puede ser dividida en agricultura para el consumo y agricultura para el mercado. Esta participación en el mercado a una mayor escala por la compra de bienes industrializados ha obligado a la población a pagar un precio elevado por las mercancías provenientes de la capital, las cuales solo llegan a través de intermediarios (Soto, 2010, pág. 10).

Finalmente Ochoa $(2007,5)$, examina el turismo como una posible nueva bonanza económica en la amazonia, enfocándolo a la perspectiva de "cadenas globales de mercancías" como un elemento mundial que ha ejercido procesos de cambio en el ámbito de lo local del trapecio. En estas interacciones, las comunidades se describen como dinámicas en su interacción con el mercado moderno mediante su participación en esta cadena. En este sentido, la lógica local se ha transformado como parte de su proceso histórico y de contacto, generando nuevas respuestas y definiciones, por lo cual hoy resulta incompleto aproximarse a un sistema económico local sin contemplar lo que sucede alrededor en un contexto más amplio de economía regional, nacional y global (Soto, 2010, pág.17).

Por tanto se observa que los cambios en las comunidades indígenas se evidencian principalmente en sus actividades socioeconómicas, distribuidas en actividades que se enfocan a la producción de diferentes elementos para la obtención de dinero, esto de la mano del creciente turismo como una fuente de empleos, y el escenario para comercializar sus artesanías y productos agrícolas.
Ahora bien, es necesario mostrar la historia de Mocagua dentro del contexto globalizador del Amazonas, para ello se retoman aportes del libro "Tomando masato en Mocagua (mucawe)" elaborado en su totalidad por Héctor León, habitante de la comunidad quien realizó este trabajo a partir de una investigación local de vida ancestral del Tikuna de Mocagua.

Mocagua en un primer momento tenía su asentamiento en la isla con el mismo nombre, ubicada en medio del río Amazonas entre la frontera de Perú y Colombia, hacia el año 1945 Mocagua por primera vez tiene presencia de policía, estos llegaron como colonizadores haciendo sus fincas y cultivos en el territorio. En este momento el Curaca se fortalece gracias a la presencia del cuerpo de policía, por otro lado el Tikuna comienza a estudiar y a formarse, el Tikuna encuentra aquí un atropello, porque se le prohíbe su dialecto, no podía hablar su propia lengua, solo el castellano, otro problema para el Tikuna: le prohíben su bebida, no le permiten hacer masato, el agente de policía miraba el cántaro lleno de masato, le volteaba el cántaro y lo botaba, esto ya era un abuso, pero nadie reclamaba los derechos que tenía el Tikuna. (León, sf, pág. 15-25).

Como puede evidenciarse los habitantes de Mocagua reconocen la llegada del cuerpo de policía el cual se instala debido al contrabando, narcotráfico presente en la zona, como una forma de represión a sus costumbres, por otro lado durante este lapso de tiempo también llega el internado San Francisco de Loretoyaco, el cual como organización religiosa prohíbe el uso de la lengua materna a los estudiantes y obliga el uso del castellano, a su vez reprime muchas de sus prácticas culturales y les aplica castigos físicos, la permanencia de este internado generó la pérdida del uso de la lengua en los habitantes de Mocagua, lo cual se mantuvo hasta tal punto que hoy en día pocas personas dentro de la comunidad manejan su idioma materno.

Después de la caída del auge del narcotráfico, el contrabando de madera y pieles, la economía está basada en la pesca, el turismo, el comercio de productos traídos de Leticia y distribuidos en tiendas locales, ingresos producidos por la fundación Maikuchiga la cual tiene como objetivo la conservación de los primates en especial del churuco, empleos formales con Parques Nacionales Naturales, la reserva privada de Calanoa, y en una menor medida de las artesanías. 
Para el año 2006 el gobierno de la época, autoriza la implementación del modelo de concesión en el Parque Nacional Natural Amacayacu, con el objetivo de crear una sede del Hotel Decameron dentro de la zona del parque traslapada con la comunidad de Mocagua. Hacia el año 2012 se cierra la concesión del hotel, el cual fue una importante fuente de empleos e ingresos económicos para la comunidad de Mocagua, durante la época del hotel el auge del turismo impulso la expansión de la comunidad así como la compra de bienes como electrodomésticos traídos desde Leticia, sin embargo también trajo problemáticas externas a la comunidad como el alcoholismo, la drogadicción y el reemplazo de prácticas como la pesca y el cultivo de la tierra por otras como la guianza de turistas, y los empleos propios del hotel.

Retomando lo anterior se evidencia como esta comunidad ha sido expuesta a dinámicas y prácticas que no son propias de su tradición como indígenas, y que han venido consolidándose por todo el trapecio amazónico, esto ha generado en la comunidad cambios en su forma de vida, en sus actividades sociales y económicas, que les han generado nuevas necesidades que antes no tenían, como incluirse en el mercado laboral y económico occidental.

En este sentido surge la iniciativa por parte de los autores de investigar: ¿Cuáles han sido las transformaciones sociales, culturales y económicas en la comunidad de Mocagua producidas por el proceso de globalización? Para ello se estructura el desarrollo de la investigación en torno a tres ejes: Identificar las características de la globalización en términos económicos, y sociales buscando la dimensión de su impacto e influencia, caracterizar las dinámicas sociales ancestrales de la comunidad para tener claridad de su proceso histórico y establecer las transformaciones de sus prácticas socioeconómicas para ser contrastadas desde el proceso de globalización.

\section{Fundamento teórico}

En el siguiente apartado se desarrolla teóricamente las categorías de análisis propuestas para la investigación, siendo estas: globalización, dinámica cultural, práctica socioeconómica eidentidad.

El presente trabajo ubicará a la globalización en la postsegunda guerra mundial, encontrando útil ubicarla en la década de los 70's por los hechos políticos y económicos que sucedían en el orden mundial, la inauguración del thatcherismo en Inglaterra en 1979, seguido por los años de gobierno de Reagan - Bush y la expansión definitiva del neo-liberalismo a la mayoría de los rincones del mundo; representando "el apogeo del capitalismo financiero, la acumulación flexible, la ideología de libre mercado, la caída del muro de Berlín, la consolidación de la sociedad de la red y el denominado nuevo orden mundial" (Escobar, 2010, pág. 189).

Con lo anteriormente expuesto, la definición que se retomara para el desarrollo de este trabajo investigativo será la de Arturo Escobar (2010) quien dice que "la globalización es caracterizada por ciertas instituciones tales como el estado - nación, y por algunos rasgos básicos tales como la auto - reflexividad (la continua retroalimentación entre el conocimiento experto y la sociedad, transformándola); el desmembramiento de la vida social del contexto local y sus crecientes determinaciones por fuerzas externas; y el distanciamiento espacio/tiempo o la separación de espacio y lugar, lo cual significa que las relaciones entre ausentes, se convierten más importantes que la interacción cara a cara" (Escobar, 2010, pág. 192).

A modo de conclusión la globalización constituye una nueva manera de pertenecer al tiempo y al espacio, haciendo una diferencia entre pasado, presente y futuro, privilegiando de manera especial a la economía del mercado y generando un cambio en la esfera pública y el autogobierno de los pueblos, fortaleciendo la idea de un proceso de globalización emanado de algunos pocos centros dominantes.

Se considera adecuado tomar lo planteado por la Teoría del control de Guillermo Bonfil Batalla, quien expone que los elementos culturales pueden ser propios o ajenos. Son elementos propios los que la unidad social considera ha recibido como patrimonio cultural heredado de generaciones anteriores y los que produce, reproduce, mantiene o transmite, según la naturaleza del elemento cultural considerado. Inversamente son elementos culturales ajenos aquellos que forman parte de la cultura que vive el grupo pero que este no ha producido ni reproducido (Bonfil, 1988, págs. 13- 53).

Retomando la teoría del control de Bonfil (1998), a continuación se mostraran los principales elementos de 
análisis para comprender las dinámicas culturales, se definirá cultura autónoma, cultura impuesta, cultura apropiada y cultura enajenada:

Cultura autónoma: en este ámbito la unidad social toma las decisiones sobre elementos culturales que son propios porque los produce o porque los conserva como patrimonio pre- existente. La autonomía de este campo de la cultura, consiste precisamente en que no hay dependencia externa en relación a los elementos culturales sobre los que se ejerce control; uno de estos por ejemplo podrían ser las prácticas curativas tradicional. Como lo plantea Quintana (2017) "Existe la particularidad de que en la comunidad se considera como enfermedad la brujería y la mala suerte. Esto podría explicarse desde la percepción de la morla y el corpus mítico que se maneja dentro de la comunidad. Es así que el origen de la enfermedad pueden ser las envidias y maleficios que se hacen a través de brujos u otros medios. Debido a esto se tiene la concepción que una persona buena, generosa, respetuosa, que sabe escuchar, aprender y enseñar no estará propenso a contraer enfermedades, a no ser que otros hayan enviado enfermedades a través de la magia"

Otros ejemplo de esto son: la agricultura tradicional: conocimientos implicados (sobre tipos de suelo de semillas, calendario agrícola, previsión del tiempo, identificación de plagas, etc.), los instrumentos agrícolas fabricados en la propia comunidad, las formas de organización del trabajo, para los momentos críticos de la labor, rituales asociados a las distintas fases del ciclo agrícola y todos los demás elementos que intervienen en el proceso completo de la agricultura, se presentan como elementos propios sobre los cuales las comunidades tradicionales ejercen decisiones propias. Las acciones de la vida doméstica serían otro ejemplo posible de componente del ámbito de la cultura autónoma (Bonfil, 1988).

En este sentido la cultura autónoma se entiende como todos aquellos elementos propios de la comunidad, que se han construido tradicionalmente y que se trasmiten generacionalmente. Estos elementos son los que construyen la identidad propia de los pueblos.

Sin embargo debido a los procesos de globalización que históricamente se han evidenciado en el trapecio amazónico han llegado muchos elementos culturales, principalmente occidentales, en este sentido se ve el surgimiento de una cultura impuesta:

Cultura impuesta: Este es el campo de la cultura etnográfica, en el que ni los elementos ni las decisiones son propios del grupo. Un ejemplo puede ser la enseñanza escolar (la escuela como institución) en muchas comunidades todas la decisiones que regulan el sistema escolar se toman en instancias ajenas a la comunidad y los elementos culturales que se ponen en juego son también ajenos. Las actividades religiosas que desarrollan misioneros de diversas iglesias caen igualmente en este ámbito, por lo menos en las etapas iniciales de la penetración, cuando el personal misionero es ajeno, los contenidos dogmáticos y las practicas rituales también y las decisiones son externas. Así mismo, la presencia de medios de comunicación externos (Bonfil, 1988).

Según lo expuesto la cultura impuesta son aquellos elementos que han sido implantados en las comunidades por agentes externos, desplazando elementos propios o creando sincretismo entre los mismos, como se ampliara en la siguiente definición:

Cultura apropiada: este ámbito se forma cuando el grupo adquiere la capacidad de decisión sobre elementos culturales ajenos y los usa en acciones que responden a decisiones propias. Los elementos continúan siendo ajenos en cuanto el grupo no adquiere también la capacidad de producirlos o reproducirlos por si mismos; por lo tanto, hay dependencia en cuanto la disponibilidad de esos elementos culturales, pero no en cuanto a las decisiones de su uso. El uso de elementos culturales ajenos implica, la asimilación y el desarrollo de ciertos conocimientos y habilidades para su manejo, la modificación de ciertas pautas de organización social o la incorporación de otras nuevas, el reajuste de aspectos simbólicos y emotivos, que permita el manejo subjetivo del elemento apropiado, son esos cambios en la cultura autónoma, los que hacen posible la formación de un campo de cultura apropiada (Bonfil, 1988).

Finalmente se definirá la cultura enajenada, este ámbito se forma con los elementos culturales que son propios del grupo, pero sobre los cuales ha perdido la capacidad de decidir: es decir, son elementos que forman parte del patrimonio cultural del grupo pero que se ponen en juego a partir de decisiones ajenas. En la 
situación de un grupo dominado, los ejemplos pueden abarcar una gama amplia de elementos culturales.

La enajenación de recursos inmateriales podría ejemplificarse cuando la folklorización de fiestas y ceremonias son usadas para su aprovechamiento turístico, sería un caso en que elementos de organización, materiales, simbólicos y emotivos propios, quedan bajo decisiones ajenas y, en consecuencia, forman parte del ámbito de la cultura enajenada" (Bonfil, 1988, págs. 13-53).

Si bien lo expuesto por Bonfil aclara en buena forma el concepto de dinámica cultural, es necesario precisar que estas dinámicas no se evidenciarán en todas las situaciones y contextos teniendo en cuenta la complejidad de la realidad social, por ello la única forma para conocer a fondo estos contextos es realizar un estudio desde la particularidad y la inmersión con las comunidades.

La dinámica cultural se puede entender como aquellos movimientos que experimenta un grupo o comunidad en sus costumbres, actividades, emocionalidades, tradiciones, y prácticas; estos pueden darse en el contacto con otras dinámicas culturales, en este caso el grupo de forma consciente o inconsciente asimila estas nuevas dinámicas donde mantiene algunas propias y pierde otras, pero también puede poner sus propias dinámicas al servicio de otros, ya sea por obligación o como una forma de obtener alguna retribución.

La nueva ola de globalización que ha recomenzado en la década de los 90's, como consecuencia del término de la guerra fría, ha estado acompañada por la emergencia de antiguas identidades locales, reafirmación de minorías nacionales, religiosas y étnicas, en todas partes del mundo. En todos los continentes y países van de la mano la globalización de los mercados de productos, servicios e informaciones y la reafirmación, a veces neurótica, violenta y no pocas veces fundamentalista, de las propias identidades, de los asuntos locales (Bengoa, 2000, págs. 34-40).

Pareciera que la vida económica depende cada vez menos del ámbito local, en cambio la vida cultural, las propias identidades, dependen de lo que se es, se ha sido y se quiere ser como comunidad. Es por ello que con sorpresa se ve aparecer cada día nuevos grupos minoritarios exigiendo sus derechos, como nuevos grupos que quieren conservar sus tradiciones, lenguas y cultura.

En América Latina la cuestión de las minorías, de las nuevas identidades, de la reaparición de las relaciones sociales primarias tiene su máxima expresión en la cuestión indígena. No es que no existan otros grupos minoritarios, sin embargo, la cuestión indígena es la que más peso ha tenido (Bengoa, 2000, págs. 34-40).

Al realizar trabajos de terreno en comunidades indígenas, la precepción de los indígenas es que la globalización se constituye en una "amenaza" cultural profunda. La transformación de las experiencias locales viene a resituar las identidades locales en un contexto radicalmente diferente, en el mundo comunitario tradicional no era necesario explicar la propia identidad; no se precisaba dar cuenta de esta ante nadie, en un mundo cada vez más interrelacionado circula la pregunta obvia: ¿y tú, quién eres? (Bengoa, 2000, págs. 34-40).

Frente a la pregunta por la identidad, los indígenas se ven presionados a iniciar un complejo proceso de respuestas, es como un juego de espejos, de preguntas y respuestas, que depende de "como nos observan" y "como observo al otro". Ante la potencial amenaza, ante el temor de no tener una propia identidad construida, ante el peligro de encontrarse y respuestas ante las preguntas de extraños, comienza a surgir una necesidad evidente por poseer un discurso de identidad.

Los recursos locales, la sabiduría tradicional no siempre son útiles para la elaboración de este nuevo discurso acerca de la propia identidad grupal, el nuevo discurso debe ser comprensible por los foráneos, requiere por lo tanto del conocimiento de sus claves culturales, de sus significados, de sus símbolos. Este nuevo discurso de las identidades étnicas tiene un camino de ida y regreso; se fundamenta en última instancia en lo que ha sido la tradición identitaria de la comunidad, la que se puede denominar como la "identidad tradicional"; el discurso viaja por las culturas adyacentes en particular por la cultura dominante que es a quien se dirigirá, allí se reprocesa, adquiere un lenguaje nuevo, diversas entonaciones e incluso cambia las prioridades como consecuencias de alianzas culturales en un tercer movimiento discursivo, vuelve a la comunidad, es 
asumido como "el nuevo discurso" y muchas veces reemplaza incluso al discurso identitario tradicional (Bengoa, 2000, págs. 34-40).

La cuestión indígena y la aparición de nuevos discursos identitarios se explica por medio del proceso de globalización cultural que se ha referido y que tiene como reacción su contraparte en la emergencia indígena.

El proceso de globalización actual, es el mayor productor de discursos de identidad indígena en distintas comunidades de Colombia, y en particular las de la rivera del río Amazonas.

En este sentido la relación entre cultura e identidad es entonces estrecha en cuanto ambas son construcciones simbólicas, pero no son la misma cosa. Mientras la cultura es una estructura de significados incorporados en formas simbólicas a través de los cuales los individuos se comunican, la identidad es un discurso o narrativa sobre sí mismo construido en la interacción con otros mediante ese patrón de significados culturales (Mead, 1974).

Mientras estudiar la cultura es estudiar las formas simbólicas, estudiar la identidad es estudiar la manera en que las formas simbólicas son movilizadas en la interacción para la construcción de una auto-imagen, de una narrativa personal. La construcción de identidad es así un proceso al mismo tiempo cultural, material y social. Cultural, porque los individuos se definen así mismos en términos de ciertas categorías compartidas, cuyo significado está culturalmente definido, tales como religión, género, clase, profesión, etnia, sexualidad, nacionalidad que contribuyen a especificar al sujeto y su sentido de identidad. Estas categorías podríamos llamarlas identidades culturales o colectivas. Es material en cuanto los seres humanos proyectan simbólicamente su sí mismo, sus propias cualidades en cosas materiales, partiendo por su propio cuerpo; se ven a sí mismos en ellas y las ven de acuerdo a su propia imagen. Es también un proceso social, porque la identidad implica una referencia a los "otros" en dos sentidos. Primero, los otros son aquellos cuyas opiniones acerca de nosotros internalizamos, cuyas expectativas se transforman en nuestras propias auto-expectativas (Mead, 1974).
Con base en lo anterior, se comprende la identidad como una construcción histórica de los pueblos indígenas, que parte de factores culturales elaborados y transmitidos generacionalmente por medio de sus prácticas, costumbres y educación propia.

Lo socioeconómico no se refiere a la suma o yuxtaposición entre los enfoques teóricos de la sociología y la economía, tampoco lo es en alguna de sus variantes, según se le asigne más importancia a una u otra de estas dos disciplinas; ni se trata de una economía con agregado de aspectos sociales (como por ejemplo, los complementos de datos estadísticos sobre variables supuestamente puramente sociales o perspectivas subjetivas, comunicativas, antropológicas o psicológicas entre otras), ni tampoco lo es en el otro sentido, como una mirada social de la realidad con agregados de términos formales de económicos y con datos cuantitativos sobre, por ejemplo, los aspectos productivos o financieros de una situación determinada (Amitai, 2007, págs. 994- 996).

Se entenderá lo socioeconómico como un enfoque teórico y necesariamente transdisciplinario, que pretende comprender integralmente la complejidad social a partir de observar describirla, analizarla para actuar en y desde la realidad. Coraggio y Arancibia (2006), lo socioeconómico no solo se abstendrá a aproximaciones segmentadas y/o fraccionadas de la realidad, apuntan a contener las herramientas necesarias para abordar la complejidad social, sino que fundamentalmente recupera en su seno a la economía, como ciencia, como método, como perspectiva de conocimiento, por considerarla parte fundamental para la acción social y clave para una comprensión de la realidad que es holística, indispensable para el conocimiento actual y potencial de las estructuras materiales de la sociedad (citado en Amitai, 2007, págs. 994-996).

Lo socioeconómico se constituye como un enfoque integrador que busca comprender la complejidad de la realidad social para actuar sobre ella, por ello se entenderán las prácticas socioeconómicas teniendo como base este enfoque.

Es así, como las prácticas socio-económicas incluyen aquellas actividades destinadas a la obtención, procesado y/o conservación de alimentos y a la 
fabricación y mantenimiento de implementos, cuyo destino originario se orientó a la satisfacción de las exigencias mínimas de la vida social: alimento y cobijo para la sociedad. Así pues, en cuanto la materia obtenida, aprovechada, transformada y/o mantenida, las condiciones materiales u objetos cobran un sentido originario en las prácticas socio-económicas (Amitai, 2007, págs. 994-996).

Las prácticas socio-económicas "se establecen entre hombres y/o mujeres e involucran y dimensionan el mundo de los objetos. Éstos son reconocidos desde la arqueología como calidad de objetos arqueológicos. Que comportan tres planos de expresión en cuanto a artefactos, arteusos y circundatos (Castro, et al., 1996, págs. 35-48).

Todas las actividades destinadas a mantener esa función de fabricación y transformación constituyen también una actividad productiva, por lo que queda englobada dentro del dominio de las prácticas socioeconómicas, lavar la ropa, barrer la casa, limpiar la vajilla doméstica o afilar un hacha son prácticas socio-económicas, en cuanto contribuyen a mantener en funcionamiento determinados productos (la ropa, la vivienda, la cerámica o el hacha), un hacha sin filo o un automóvil sin carburante pierden su plano artefactual, a menos que sean re-producidos (reciclados), es decir, utilizados en otra función diferente a la desempeñada originalmente, como, por ejemplo, la reconversión del hacha en martillo o del automóvil en refugio" (Castro, et al., 1996).

De este modo la esfera de las prácticas socioeconómicas incluye el mantenimiento de las condiciones materiales producidas y, eventualmente, las operaciones necesarias para su desecho, resulta conveniente recalcar la importancia del mantenimiento como uno de los momentos de la producción que, desde esta perspectiva, pasa a dotarse de una dimensión que podría llamarse de continuidad. La producción no finaliza a la salida del taller (como generalmente se tiende a asumir), sino que se prolonga hasta la amortización (desecho) del artefacto. Esta constatación abre también la posibilidad de analizar fenómenos de explotación económica frecuentemente pasados por alto, ya que el mantenimiento de los artefactos exige una inversión de trabajo que, en ocasiones, puede exceder incluso la que los conformó como tales, entre estos trabajos figuran en lugar destacado las llamadas "labores domésticas" (limpiar, lavar, barrer, etc.), cuyo peso en la actividad económica es a menudo desestimado en favor de la 'macroeconomía" (o "economía política"), cuando, en realidad, aquéllas son en última instancia parte integrante de la infraestructura de ésta.

Para ampliar lo anterior se refiere que el plano de los arteusos hace alusión a materia beneficiada para el consumo alimentario o bien materia apropiada o desechada para la consecución de artefactos. En el primer caso figuran elementos botánicos, faunísticos o minerales (granos. frutos. partes de animales, agua o sal). Los segundos se muestran como materia orgánica o inorgánica descontextualizada de su matriz geológica (mineral metálico, sílex. arcilla) o biológica (troncos, fibras vegetales), o bien como residuos de la materia transformada por la mediación de artefactos (escorias. lascas, carbones de hogares). Si bien los artefactos siempre se encuadran dentro de la categoría de producto, únicamente los arteusos de tipo alimentario y las materias primas destinadas a la fabricación de artefactos son susceptibles de ser incluidos en aquélla. En lo que respecta a la producción alimentaria, sólo se ha tenido en cuenta, en general, la obtención del producto primario, por ejemplo la cebada o la carne (Castro, et al., 1996, págs. 35-48).

"Sin embargo, se han dejado de lado aquellas actividades relacionadas con la transformación del producto (molienda, cocinado, extracción del veneno de la yuca, despellejamiento, descuartizamiento, etc.) o la conservación o el almacenamiento del mismo (salazón, ahumado, torrefacción), en el que se incluye el control higiénico de los contenedores para evitar la putrefacción, la acción de roedores o insectos, etc" (Castro, et al., 1996).

Finalmente, el plano de los circundatos conlleva a la presencia de elementos naturales constitutivos del objeto en cuanto a indicadores de las condiciones medioambientales del espacio natural. Sin embargo, la noción de espacio natural resulta ambigua en lo que respecta a las prácticas sociales. Utilizar el término de espacio social, que constituye la esfera física relacional de la apropiación de la materia por parte de hombres y mujeres. Este concepto integra de manera dinámica las dimensiones de espacio natural y territorio, entendido 
éste como la articulación y/o gestión (ordenada / planificada o no) de la materialidad que las prácticas sociales exigen. El territorio supone una organización del espacio generada por la reproducción económica y política de un grupo social, de forma que supone una antropización del espacio natural. El territorio presenta diversas dimensiones, como mediador de información, espacio de subsistencia y espacio político de cohesión o coerción. Por otro lado, el universo simbólico con que la sociedad percibe el espacio social y el espacio natural configura el paisaje social (Castro, et al., 1996, págs. 3548).

Para el desarrollo de esta investigación las prácticas socioeconómicas estarán basadas en la definición de la Liga de Defensa por el Medio Ambiente (LIDEMA) "aquellas que comprenden los servicios y recursos naturales, las actividades y el acceso a ellos que requiere un individuo, un hogar, una familia o una comunidad para vivir bien, es decir vivir en armonía entre las personas, en equilibrio con la Naturaleza y espacios territoriales donde se exprese la identidad y se cultive la creatividad". (LIDEMA, 2011, pág. 6).

Buscando una visión clara y precisa acerca de la posesión, tenencia, propiedad, concentración, distribución y disponibilidad de las tierras; el uso y aprovechamiento de las que estuvieren ocupando y el cumplimiento de la función social de la propiedad en las tierras de resguardo, conforme a los usos, costumbres y cultura de la respectiva comunidad; la calidad, condiciones y uso de los suelos; el tamaño y distribución de la población, su situación socioeconómica y cultural; la infraestructura básica existente, y la identificación de los principales problemas.

Este planteamiento se escoge ya que desarrolla puntos clave como son: la relación del individuo, la familia y la comunidad con la naturaleza y el territorio, los cuales son puntos fundamentales de análisis cuando se abordan comunidades indígenas, y son el territorio y la naturaleza los principales puntos de tensión cuando se aborda un análisis desde los procesos de globalización.

\section{Materiales y métodos}

\section{Ingreso a la comunidad}

El desarrollo de la investigación se ejecuta desde la propuesta metodológica etnográfica planteada por
Bizquera, se desea mostrar en este artículo cómo se realizó el paso a paso de la investigación, aplicando la teoría al campo de acción, contextualizando en primer lugar la ubicación y algunos datos relevantes del resguardo.

El resguardo de Mocagua se encuentra traslapado con el Parque Nacional Natural Amacayacu, ubicado a $65 \mathrm{~km}$ de la ciudad de Leticia, donde la única forma de llegar es por vía fluvial, recorriendo el río Amazonas. Según el censo del año 2007 Mocagua posee una población de 458 personas, donde el 55\% son hombres y el $45 \%$ mujeres. (Asociación de Cabildos Indígenas del Trapecio Amazónico, 2010). Dentro del resguardo solo existe una escuela de básica primaria, donde cuentan con grupo de etno-educadores, cuando los niños son promovidos a secundaria, deben dirigirse a la población vecina de Macedonia, la cual se ubica aproximadamente a 20 minutos río abajo. Así mismo existen dos estaciones claramente diferenciadas: aguas altas y aguas bajas, las cuales condicionan la actividad natural y antrópica. Los ríos de la región fluctúan en caudal y en el nivel de las aguas drásticamente durante el año, donde el mes de septiembre (temporada seca) las playas quedan al descubierto y conforme aumentan las lluvias (mayo), el nivel del río alcanza cerca de 10 metros de inundación, en un área de al menos 500 metros de bosque inundable o várzea en las riberas del río Amazonas y sus quebradas afluentes. Por esta razón, la visita al Amazonas es drásticamente diferente en las dos temporadas y para entender la grandeza de este ecosistema es necesario visitarlo como mínimo en dos ocasiones.

Los bosques tropicales de baja altitud como los del Parque Amacayacu albergan una alta diversidad de especies y aunque tienen un bajo endemismo, poseen rangos de distribución pequeños y nichos altamente especializados.

Bizquera (2004) plantea unas etapas en el proceso etnográfico, en primer lugar el diseño, este se considera flexible y no necesariamente preconcebido. En la segunda etapa definida como la determinación de técnicas, se dio un valor especialmente importante a la observación participante, el autor Bizquera, la define como una estrategia de recolección de datos, sin embargo esta estrategia también permite establecer relaciones de cercanía y confianza con las personas de la comunidad, que hace más fácil la posterior aplicación 
de otras técnicas complementarias como las entrevistas informales, los grupos focales y los diálogos informales. La elaboración de los instrumentos propiamente dichos, surge del proceso de operacionalización de categorías: globalización, práctica socioeconómica, dinámica cultural e identidad. Cabe anotar que al llegar al contexto e interactuar con la comunidad surgen más interrogantes, los cuales son acoplados, paulatinamente a los instrumentos y actividades propuestas.

La tercera etapa denominada como el acceso al ámbito de investigación, se realizó mediante un proceso previo de consultas y de gestión con la institución y la comunidad las cuales iniciaron a principios del año 2015. El equipo de investigadores realizó numerosos contactos con varios funcionaros de la Regional Amazonía de Parques Naturales, y el PNN Amacayacu, por medio de sus experiencias y conocimiento se fue creando una idea de la comunidad. Gracias a esta gestión se logra el encuentro con la comunidad, a mediados del año 2015, donde se socializa la propuesta de investigación y la invitación a su participación activa. Este contacto inicial con la población fue clave para generar lazos de confianza y apoyo con la comunidad.

En este primer encuentro con la comunidad se socializa el proyecto, detallando las técnicas a usar, los objetivos y los resultados esperados, también haciendo claridades como el carácter no lucrativo de la investigación, los días de permanencia en la comunidad y la participación voluntaria de cualquier persona que desee contribuir al proyecto. Después de lograr acuerdos con la comunidad, queda programado el inicio del trabajo de campo.

Una vez da inicio el trabajo el equipo se reúne con la comunidad para encontrarse con el curaca, allí es recibido calurosamente por la comunidad, y se programa una asamblea para recordar los compromisos y desarrollar las dudas que hayan surgido.

Este momento marca el inicio de la cuarta etapa del proceso metodológico, definida como la selección de informantes, desde la primera asamblea se buscó entablar relaciones abiertas con diferentes personas. La estrategia adoptada por el equipo de investigadores fue la de acercarse a las personas en los espacios de actividades comunitarias, especialmente los partidos de fútbol, donde después de una charla se concretaban visitas a sus hogares para ampliar información de algún un tema en particular, almorzar, tomar café, conocer más de sus ocupaciones; esto permitió conseguir el Rapport $^{3}$ con varias personas y sus familias, las cuales posteriormente hacían parte de sus eventos y reuniones familiares al equipo de investigadores.

En este apartado se destaca como aprendizaje adquirido para el equipo de investigadores, que las competencias personales son vitales para el proceso, en cuanto se debe tener una mentalidad abierta, libre de juicios, receptividad y sobre todo humildad, elementos indispensables si se desea conseguir el rapport con las familias y la comunidad en general. En este sentido Taylor y Bogdan (Ortiz, 2015) proponen algunas estrategias para el investigador etnográfico a fin de conseguir el rapport, estas son: acomodarse a las rutinas y maneras de hacer las cosas de los participantes; recurrir a aquello que se tiene en común; ayudarles; adoptar una postura humilde; y demostrar el interés por la información que transmiten. A lo largo de este acercamiento también se irán seleccionando aquellas personas a interrogar y las situaciones que se desea examinar más en detalle apropósito e intencionalmente (Ortiz, 2015).

\section{Recolectando la información; compartiendo con la comunidad}

El proceso de recolección de datos se inició en la comunidad de Mocagua, una vez el equipo de investigadores conoció el contexto, e identificó a las personas influyentes dentro de la comunidad. Esto fue acordado previamente con la comunidad, en una asamblea convocada por el curaca y varios líderes, donde el equipo de investigadores explicó detalladamente el proceso, sensibilizando a la comunidad e invitándolos a participar de forma voluntaria. Posteriormente al momento del ingreso nuevamente se convocó a una asamblea para dar inicio formalmente al trabajo de campo.

Privilegiando la observación participante en un lapso de tiempo de tres meses, se buscó gestionar con diferentes personas en la comunidad la participación del equipo en diferentes actividades de su vida cotidiana, eventos especiales y actividades.

El equipo estuvo en días de pesca, cultivo de la chagra, asambleas comunitarias, festividades, días deportivos, 
recorridos que los miembros de la comunidad venden a los turistas, labores del hogar, eventos de la comunidad religiosa etc.

Por otro lado se realizaron dos grupos focales, el primero con los adultos mayores ${ }^{4}$ de la comunidad, este grupo se desarrolló alrededor de una taza de café en el hogar que uno de ellos facilitó para el encuentro. El segundo con los más jóvenes de la comunidad ${ }^{5}$.

Finalmente se recurrió a la aplicación de entrevistas semiestructuradas, las cuales fueron focalizadas a las personas que tienen mayor influencia dentro de la comunidad, o son las más reconocidas por su labor, actividad económica o participación activa en los procesos comunitarios. Dentro de estas personas encontramos mujeres, que desempeñan no solo su rol en el hogar sino también en la elaboración de artesanías y el comercio de productos, se contó también con el aporte del curaca de la comunidad, el director de la escuela, un miembro de la guardia indígena, un habitante que a su vez es funcionario del Parque Nacional Natural Amacayacu.

Estas entrevistas fueron desarrolladas participando en las labores o tareas de las personas, por ejemplo se realizó la entrevista a las artesanas mientras elaboraban canastos de chambira (Astrocaryum chambira), en las jornadas de pesca, y mientras se cultivaba la chagra; de esta forma el equipo de investigadores aprendió a pescar, a cultivar la tierra, a elaborar canastos en chambira, lo que generó una mayor aceptación por parte de la comunidad, y facilitó en gran medida el diálogo y la obtención de la información.

El diálogo informal permanente con los habitantes de la comunidad fue fundamental, permitió realizarse en cualquier momento, aportando datos valiosos y permitía verificar y contrastar la información proporcionada por otras personas, lo cual era plasmado en los diarios de campo.

\section{Hallazgos}

A continuación se muestran los hallazgos que el equipo de investigadores encontró con referencia a cada una de las categorías de análisis propuestas. Estos se redactan como un relato donde se da voz a las personas que fueron entrevistadas, y a las experiencias de los investigadores en el trabajo de campo, obtenidas gracias a la interacción con la comunidad, donde se asistió a días de pesca, largas caminatas, mingas y celebraciones, donde las personas abrieron las puertas de sus hogares e invitaron a los investigadores a ser parte de su cotidianidad.

Se da inicio abordando el tema de la pérdida de costumbres desde la primera categoría Globalización; los Tikuna tradicionalmente poseen un conjunto de costumbres propias de su etnia, las cuales surgen de su historia, su mitología y la relación misma con la naturaleza. La familia Tikuna originalmente se constituía a partir de acuerdos entre los diferentes clanes (paujil, arriera, águila, garza, etc) en donde las personas de un clan solo pueden casarse o formar parejas con otra de un clan específico; sin embargo en la actualidad esto difícilmente se cumple, en este sentido expresa una habitante de Mocagua: "ahora nosotros regalamos los hijos como un gajo de plátano, porque ya no es como antes, que era muy sagrado, algo muy delicado de hacer eso". (Mocagua M. d., 2015, cod. Ep2s2), aquí expresa el significado sagrado de los hijos, el cual esta persona considera se ha perdido con el tiempo.

Uno de los eventos más importantes para los Tikuna es la pelazón. Este es un ritual el cual se desarrolla cuando a las niñas de la comunidad les llega su primer periodo menstrual. Frente a esto se observa que en Mocagua ya no se celebra este ritual, expresa uno de sus habitantes "desde el noventa para acá nos vinimos colgando, nos colgamos, ya la gente no quería, los niños tampoco, los padres eso que le importaban" (Mocagua D. e., 2016, cod. Ep3-s3), esto muestra como el abandono de este ritual no fue algo que decidió la comunidad, sino más bien algo que se fue perdiendo y progresivamente dejo de ser parte de su costumbre.

Frente a la estructura de gobierno Tikuna se han evidenciado cambios notorios, antiguamente la máxima autoridad para los Tikuna era el chamán, aquella persona conocedora de los secretos de la selva y quien podía comunicarse con la naturaleza y sus espíritus. En el proceso de globalización de la Amazonía, el Estado Colombiano reguló la organización política de las comunidades indígenas, introduciendo la figura del cabildo encabezado por el curaca ${ }^{6}$ así como también la figura de la democracia representativa. Para Mocagua

"o como ellos mismos se hacen llamar "ancianos o abuelos"

${ }^{5}$ En el desarrollo de esta actividad, los investigadores se acoplaron a una actividad deportiva planteada por la comunidad.

${ }^{6}$ El máximo cargo dentro de la comunidad y por ende la máxima autoridad, el curaca es elegido anualmente en Mocagua por votación, junto con su cabildo y su función es la de representar a la comunidad frente al Estado y las otras comunidades, además de cumplir labores administrativas y de gobierno interno de la comunidad. 
esto se materializa en la resolución 060 de 19837.

Durante la historia de la comunidad han existido grandes bonanzas, las más recordadas por los habitantes son la extracción del caucho, la extracción de madera, las pieles, el narcotráfico y el turismo; esta última aunque ha disminuido su auge aún representa la actividad económica principal para una buena parte de la comunidad. La concesión del hotel Decamerón dio inicio hacia el año 2006, esta se realizó con el PNN Amacayacu, y benefició directamente a la comunidad de Mocagua.

Esto disparo la circulación del dinero en la comunidad, ahora las personas podían ir a Leticia a comprar electrodomésticos como televisores, plantas eléctricas, neveras, mejoras para sus hogares, joyas etc... fue una época de bonanza: "Cuando teníamos al Decameron teníamos la bonaza del turismo hay dependíamos como 30 familias", "Mocagua no avanzaba, lento lento subía, pero cuando llegó el tiempo del turismo ahí si Mocagua creció, Mocagua extendió mucho". (Curaca, 2015,cod.Ea4-s4) Sin embargo las bonanzas no son eternas, en el año 2012 el Amazonas vivió un invierno sin precedente, lo cual generó que el río Amazonas y sus afluentes crecieran por encima del nivel histórico, el hotel fue inundado por las aguas del amazonas y se vio obligado a cerrar sus puertas, como lo expresa una persona quien trabajo en él: "se acabó en el 2012 chao pescao, se cerró el Decameron, no quiso abrir de ahi para acá hemos decaído mucho, decayó la comunidad" (Curaca, 2015,cod.Ea4-s4), el cierre del hotel generó una crisis en la comunidad, ahora las personas estaban desempleadas, no había forma de vender sus productos "la gente no hacia mermelada antes si se hacía, pero ¿a quién se lo vendían?, porque en Mocagua la gente estaba quebrada" (Habitante, 2016,cod.Ea5-s5) sumado a esta crisis, el turismo dejo atrás muchos problemas ajenos a la comunidad, el alcoholismo, el consumo de drogas entre otros; "La comunidad de Mocagua ha sido muy dependiente, eso generó un impacto muy negativo y la plática se movía fue por eso, entonces muchas familias bajo sus recursos, llegaron también a salirse de la comunidad y buscar empleos en otros lugares" (Habitante, 2016.cod.Ea5-s5), muchas personas al no encontrar una forma de recuperar su ingreso económico decidieron ir a Leticia a buscar empleo en el sector turístico, muchos hoy en día trabajan con el hotel Decameron que ahora funciona en esta ciudad, otros sin embargo se quedaron en la comunidad y se vieron obligados a buscar otras fuentes de sustento, esto dicho en palabras de Galeano "El dinero vuela a la velocidad de la luz: ayer estaba allá, hoy está aquí, mañana quién sabe, y todo trabajador es un desempleado en potencia" (Galeano 2005, 2). La situación se agravo ya que muchas personas al dedicarse al turismo dejaron de trabajar en sus chagras, de producir su propio alimento, y ahora debían comenzar de cero.

Una vez abordado el tema del turismo, inevitablemente surge el interrogante ¿Qué significa el dinero para esta comunidad indígena?, el dinero resulta ser un agente presente en todas las dinámicas del trapecio amazónico, este comenzó a manejarse prácticamente desde la llegada del hombre blanco, reemplazando formas tradicionales de comercio e intercambio. El dinero enmarca prácticamente todas las dinámicas de la comunidad, pero también ha traído una serie de dificultades para las personas, esto expresado en la frase "de que nos ha servido tanta plata que no se ha podido manejar y lo que ha hecho es traer daño el tema del alcoholismo, la drogadicción otras cosas el tema de las muchachas las embarazan las dejan botando ya todo eso genera un conflicto" (Habitante, 2016,cod.Ea5-s5), esto hace referencia a problemas actuales que vive la comunidad, problemas que no tenían tradicionalmente y que se han arraigado desde el contacto con occidente.

A partir de esto se evidencia como el dinero como tal se convirtió en una necesidad, y las personas buscan conseguirlo de diferentes formas, esta discusión generó en las mismas personas una reflexión frente al tema: "entonces el valor que ha generado la plata ha sido frustrante para el tema cultural, reemplaza bastante a muchas cosas y eso es como una amenaza para nosotros realmente, el no saber interpretar ese valor del dinero que tiene en estos momentos, todo el mundo está pensando que esa es la solución, itener plata mucha plata!" (Habitante, 2016,cod.Ea5-s5), "En este momento la gente piensa que el dinero está cambiando todo, que tiene un valor tan fuerte que no tiene comparación", (Curaca, 2015.cod.Ea4-s4).

Por otro lado las investigaciones en la región amazónica son comunes, la mayoría de estas se enfocan estudios sobre fauna y flora, estudios ambientales, hidrográficos y unos cuantos de tipo social, etc., Mocagua ha sido sede de muchos de estos estudios, en los cuales los

${ }^{7}$ La resolución 060 de 21 de septiembre de 1983 es un acto de gobierno donde se reconoce a Mocagua como resguardo indígena, estructurando su gobierno interno y las normas que rigen al resguardo. 
investigadores contratan los servicios de guianza, hospedaje y alimentación tal y como se observa en la siguiente frase: "yo coloco mi tarifa, mi tarifa es $\$ 40.000$ pesos el día” esa era la tarifa, la cual se manejaba para el tema de investigaciones que traían recursos" (Curaca, 2015,cod.Ea4-s4) lo anterior responde a una forma de ingreso económico que implica dejar que se hagan estudios dentro del territorio de la comunidad: "Cuando se habla de investigación, la gente aquí lo interpreta como plata, empecemos por ahí, es un tema de que la gente cree que todos los que llegan acá traen billete como dice el dicho" (Panduro, 2016,cod.Ea5-s5), esta fue una dinámica que no fue ajena al presente proyecto de investigación, incluso antes del trabajo de campo se recomendó al equipo de investigadores hacer claridad frente al tema de los recursos una vez se tuviera contacto con la comunidad, y ser claros frente al tema de que no habría una ganancia económica para la comunidad ni tampoco para los investigadores. También las personas de la comunidad manifiestan los problemas que genera el uso del dinero: "La gente en realidad si sabe en qué la va a invertir, como también hay otras que le invierten, en bebidas, en borracheras eso ha traído inconvenientes", "pero cuando ya comienzas a hablarle de plata la gente le ve de otra manera y esa una realidad en la comunidad". (Habitante, 2016,cod.Ea5-s5).

\section{Practicas socioeconómicas de la comunidad, antes y después}

Entendiendo las prácticas socio - económicas como aquellas actividades destinadas a la obtención, procesado o conservación de alimentos y a la fabricación y mantenimiento de implementos, cuyo destino originario se orientó a la satisfacción de las exigencias mínimas de la vida social: alimento y cobijo para los agentes sociales, dentro del trabajo de campo realizado con la comunidad de Mocagua se encuentran las siguientes:

En las practicas que se mantienen, los habitantes del resguardo han pasado por varios momentos "Más que todo la costumbre era la agricultura, sembrar yuca, maíz, plátano chontaduro, pan del árbol, piña, eso era lo que comíamos nosotros, también el caldo de pescado con fariña, hacíamos chicha del pan de árbol, de la yuca" (Mujer, 2015,cod.Ep1-s1) actividades que a pesar de la ola globalizante se conservan, manteniendo de cierta forma las tradiciones propias de los indígenas Tikuna. Al momento de referirse a actividades que estén destinadas a sobrevivir y supervivir se encuentra que actualmente la comunidad ha mantenido gran parte de sus actividades tradicionales, la mayoría de estas relacionadas con el obtención de comida, un claro ejemplo es la chagra la cual es la tierra para cultivo, en la chagra las personas siembran frutas, tubérculos y todas aquellas plantas que les brindan alimento y elementos para fabricar artesanías. Las chagras se construyen fuera de la comunidad, y para llegar a ellas es necesario caminar un par de horas por la selva, o en canoa por la quebrada, esto responde a una de las problemáticas actuales de la comunidad que son los robos de los productos, principalmente la yuca y el plátano los cuales tienen un valor económico y alimenticio importante para las personas de la comunidad.

El uso de los recursos naturales se ha transformado, "antiguamente se cortaba la palma, se cortaban los arbolitos para hacer los techos, porque antiguamente todo era hecho de madera redonda" (Director, 2016,cod.Ep3-s3), esto cambió cuando el gobierno nacional realiza la donación de casas para la comunidad, estas casas tienen techos con tejas de zinc, y se construyen de madera. Hoy en día se continúa extrayendo recursos de la selva, principalmente la madera para uso doméstico, la pesca y la chagra siguen siendo una práctica vital para la comunidad que no solamente asegura su comida diaria sino también un ingreso en la venta de estos. Otra forma de ingresos para la comunidad aunque en menor medida es la elaboración y comercialización de artesanías, las cuales se divide en productos de barro, yamchama ${ }^{8}$, chambira ${ }^{9}$ y madera.

Actualmente una de las principales fuentes económicas de Mocagua es el turismo ${ }^{10}$, en este sentido la comunidad cuenta con personas que ofrecen diferentes servicios a los turistas, estos son la pesca artesanal, avistamiento de delfines y de aves, caminatas en la isla, recorridos en la selva, visitas a Puerto Nariño y los lagos de Tarapoto; estas actividades varían de precio desde los 60 mil pesos hasta los 150 mil, también algunos abuelos obtienen ingresos contando cuentos tradicionales a los turistas. Uno de sus habitantes expresa "Por el momento

${ }^{8}$ Fibra extraída del árbol del mismo nombre, antiguamente se usaba para la fabricación de ropa y elementos para dibujar.

${ }^{9}$ Fibra de la palma del mismo nombre, se usa para elaborar cestos, cernidores y otras artesanías y herramientas.

${ }^{10}$ Si bien el bosque provee gran cantidad de insumos para sus actividades económicas, cabe resaltar que existen limitaciones de caza, pesca y tala de árboles, además el traslape con el Parque Nacional Natural, limita y regula el uso de los recursos que posee la selva. 
contamos con el centro privado la reserva privada de Calanoa, de ahí dependen como siete familias más o menos, del resto son ya emprendedores de aquí de la comunidad, que sacan cosas como Yegune, Tomacache ${ }^{11}$, que le generan ingresos a la comunidad", (Curaca, 2015,cod.E4-s4).

Las transformaciones a lo largo del tiempo no solo fueron en el sector económico, en el social también se pueden apreciar grandes cambios, el más llamativo es la transformación en el rol y funciones de las mujeres de la comunidad. Antiguamente los trabajos asignados a la mujer estaban bien definidos, por ejemplo dentro del trabajo de la chagra el hombre se encargaba de "tumbar y rosar" el monte, de hacer el trabajo más pesado, mientras la mujer sembraba las semillas de acuerdo a la fase de la luna, en el hogar los hombres no hacían cosas de mujer, como cocinar, lavar o atender a los niños, a la mujer se le encomendaba la crianza de las niñas, las tareas propias de la mujer, un ejemplo de esto se expresa en la siguiente frase: "si por ejemplo eran cinco hombres los cinco salían a pescar y las mujeres nos encargábamos de buscar leña, cultivar la chagra, criar a los hijos, y educar a las niñas, que como tienen que cuidarse, como tenían que remendar la ropa del esposo, que como tienen que criar a su hijo" (Mujer, 2015, cod.Ep2-s2), esto mostrando la educación impartida a las mujeres, y para los hombres se expresa en la siguiente forma: "Pues el trabajo y decirle, usted como hijo hombre, debes conducir a construir canoa, vamos a construir chagra, construir material de pesca porque el día que vas a conseguir mujer, tu mujer no va a venir a comer tierra, tiene que llevarle después que cumpla los dieciocho años jovencito debes tener casita, porque usted no va estar metido al cuarto en mi casa", (Director, 2016,cod.Ep3s3).

Esta formación en el hogar aún mantiene algunos de sus matices, sin embargo muchas de las tareas que pertenecían exclusivamente a los hombres ahora también son realizadas por mujeres, "Junto con su mujer trabajaba, por igual. Cuando hace su chagra juntos trabajaba" (Mujer, 2015,cod.Ep2-s2), ahora las mujeres van a la chagra, a tumbar árboles, porque saben manejar las motosierras y trabajan en igualdad de condiciones con los hombres. Sin embargo las funciones de las mujeres son raramente asumidas por los hombres, aunque un entrevistado manifiesta que: "aquí nadie es machista. Aquí todos nos colaboramos, por ejemplo yo aquí si veo que mi mujer está lavando pues yo tengo que hacer el desayuno, la idea es colaborarles porque no se puede echar toda la carga a una sola persona”, (Curaca, 2015,cod.Ea4-s4) la observación realizada en diferentes hogares muestra como las tareas domésticas son asumidas en su totalidad por las mujeres, lo que les genera la carga del trabajo en la chagra, la pesca más las funciones del hogar y la crianza.

Dentro de la comunidad la mayoría de familias son de tipo nuclear, ya que culturalmente los hijos al conseguir su esposa deben abandonar el hogar de los padres, por ello no se evidencio ninguna familia de tipo extensa, al menos dentro de los Tikuna que habitan la comunidad, existen padres solteros o madres solteras dentro de la comunidad frente a ello no existe ningún tipo de estigma o castigo social, se refieren esto de la siguiente forma: "si se queda por naturaleza viudo, de pronto la mujer se va, aqui se le ve es dependiendo quien tuvo el error" (Curaca, 2015,cod.Ea4-s4).

\section{El deporte transformado en un motor económico}

Efectivamente el deporte, específicamente el futbol y el microfútbol son actividades que se realizan a diario en la comunidad, no importa el clima ni la fecha, el futbol es "sagrado", y aunque es una práctica sana para jóvenes y a adultos de la comunidad este se ha convertido en uno de los principales motores económicos de la comunidad y esto se debe a que se realizan apuestas entorno a cada partido.

El fenómeno de las apuestas alrededor del fútbol es una de las principales actividades económicas de la comunidad y que se mantiene más estable, el dinero de estas en su mayoría proviene de las personas que son empleadas en las instituciones como Parque Nacional Natural Amacayacu, Reserva Privada Calanoa, Fundación Maikuchiga, Tiendas y empleos independientes, después ese dinero se mueve dentro de la comunidad hasta el momento final que este va a la ciudad de Leticia.

El hallazgo de este fenómeno se dio gracias a la convivencia en la comunidad del equipo de investigadores, los cuales centraron su observación en todas aquellas actividades desarrolladas entorno a los partidos de fútbol, se encontró que los partidos de microfútbol son espacios que muchas familias aprovechan para generar ingresos, directamente por 
medio de las apuestas, de la comercialización de productos que elaboran o que compran en Leticia $u$ otras comunidades. Por otro lado las familias aprovechan estos eventos inter comunidades para intercambiar productos e información, así como la preparación de nuevas actividades. Dentro de las gradas se ven personas de la comunidad que venden productos como: puriche, galletas, empanadas, refrescos, etc, otras personas llevan masato y chicha que elaboran en sus hogares para consumir durante el evento. Alrededor de las canchas funcionan diferentes tiendas y bares que expenden bebidas alcohólicas y demás productos.

Esta actividad como ingreso económico es reconocida ampliamente en la comunidad tal y como expresa uno de sus habitantes: "Entre familias intercambiamos a veces vendemos también pero mayormente el tema de vender es una forma de que el muchacho que va a pescar vende sus sabalitos para hacer su apuesta" (Habitante, 2016,cod.Ea5-s5).

Inicialmente la dinámica del fútbol no giraba en torno a la apuesta de dinero como tal, sino más bien de comida como lo expresa un entrevistado: "el equipo dominante ganaba la carne, la comida, la fruta pero ya el tema de dinero fue hace muy poco 1996 a 1997 más o menos fue como estructurando 2004, 2005 la concesión 2006 se hace la concesión más ingreso más gente más visitantes entonces la gente no tenía mucho en que gastar y le dedico un poco más al deporte" (Panduro, 2016,cod.Ea5-s5), y desde el cierre de la concesión se mantuvo la dinámica de la apuesta de dinero, aunque no se tienen cálculos oficiales del dinero que se mueve a diario, un entrevistado nos da una aproximación según su experiencia y es de aproximadamente 200 mil pesos por tarde, teniendo en cuenta un promedio de cinco partidos; "si una fuente de ingresos, convertido en este momento el deporte" (Habitante, 2016,cod.Ea5-s5).

Realizando una indagación más profunda frente al tema, se encuentra que el dedicar tiempo de las tardes al futbol ha desplazado otras actividades principalmente las labores de la tierra: "la familia en las tardes se ganan 34 mil pesos cualquier familia, pero se pierde el tema del plátano de la yuca entonces la gente la necesidad de ir a comprar eso va buscar a abuelas que cultivan eso", (Habitante, 2016,cod.Ea5-s5) y es algo ampliamente reconocido por algunas personas como se puede observar: "en la actualidad en la comunidad, está perdiendo un poco el tema de la actividad en la chagra en Mocagua como tal vez porque la rutina ha cambiado, antes en las tardes y las mañanas era utilizado para las chagras y ahora las tardes se utilizan en deporte" (Habitante, 2016, cod.Ea5-s5).

Esta nueva práctica socioeconómica de la comunidad replica el ciclo del dinero, ya que el dinero obtenido en los trabajos formales y de la tierra se apuesta, lo que se gana se invierte en comprar productos de las tiendas, que a su vez viajan a Leticia para surtir sus negocios.

Este espacio también es aprovechado para el intercambio social, las familias y vecinos comparten una charla, el curaca puede hacer anuncios e invitaciones a eventos como las miagas, asambleas, actividades de interés etc.

\section{Sobre las transformaciones culturales}

Finalmente se abordaran los hallazgos encontrados frente a todas aquellas transformaciones culturales que se identificaron, retomando la teoría del control cultural desarrollada por Guillermo Bonfil (Bonfil, 1988) desde los elementos de la cultura autónoma, cultura impuesta, cultura apropiada y cultura enajenada.

En Mocagua pueden identificarse elementos autónomos como el trabajo de la chagra, la cual ha sido la base de la supervivencia de los Tikuna, en esta se mantiene la forma de sembrar, la forma de recolectar y de procesar aquello que produce la tierra y que han sido conocimientos transmitidos de generación en generación. Por otro lado la división de labores entre hombres y mujeres, en donde los patrones no han variado considerablemente en el transcurso del tiempo, estos abarcan las labores en la chagra así como también las funciones domesticas en el hogar. Las mingas y la pelazón expresadas en términos de una habitante de la comunidad: "Se hacía pelazón, se hacían mingas, mi finado papá hacia mingas y la gente se emborrachaba, después de la minga pues le decían váyanse, esa era la cultura de mi finado, después de la minga se hacía un baile porque era puro acordeón que bailaba y guitarra y cantaba, esa era la cultura de finado papá”.

Mocagua se ha visto en un proceso de imposición cultural constante y permanente, un ejemplo de esto fueron las misiones religiosas que llegaron a la comunidad, y que por medio de la educación no 
solamente implantaron rituales judeocristianos sino también trataron de eliminar los rituales propios de la tradición Tikuna. Otro claro ejemplo de esto fue la escolarización bajo el modelo estandarizado del gobierno, en donde no se enseñaban las tradiciones, costumbres ni la lengua, por el contrario solo se reproducían contenidos del modelo educativo eurocéntrico que domina al país y la enseñanza del español como única opción. En este campo fue tan grande la influencia religiosa que las personas consideran la semana santa como una de sus costumbres tradicionales originales, como lo muestra la siguiente expresión: "Era difícil escuchar de alguien que tuviera una fiesta de cumpleaños, solo era la pelazón, nosotros éramos de la forma de vivir como los salvajes no sabíamos que era fiesta, lo único que si guardábamos era la semana santa, pues solo era la pelazón y la semana santa".

Se evidencian otros elementos apropiados de la cultura occidental como el reconocimiento de los derechos de las mujeres: "Eso no es como antes, ahora las decisiones son un diálogo de pareja, decidimos los dos, antes si, solo el hombre porque había un pensamiento muy machista, las mujeres tenían pocos derechos ahora tienen más derechos como nosotros y hay que valorarlas mucho", en este caso se reconoce el rol de la mujer dentro de la comunidad y se le brinda más participación. La cultura del consumo también ha sido apropiada por la comunidad, hoy en día todas las actividades de la comunidad buscan la obtención de dinero, el proceso histórico ha generado que la comunidad desarrolle nuevas necesidades que no son propias de su cultura, por ejemplo la necesidad de gastar dinero para comprar tecnología, especialmente televisores; y con la compra del TV hay que contratar el servicio de televisión satelital de Directv, ahora para comunicarse debe comprarse el celular, el cual debe tener cámara y conexión de datos, también se evidencia la compra de ropa de marcas reconocidas, de accesorios y joyas ${ }^{12}$. También debe reconocerse que se han apropiado problemáticas: "Problemas que se han visto, el tema del alcoholismo, drogadicción se ha visto, entonces la gente con plata pues a veces comienza a crearse otras necesidades, como el tabaco, drogas, también el desborono de la familia llego también como desbaratarse los núcleos familiares" patrones culturales como el alcoholismo, las drogas e incluso la violencia intrafamiliar.
Finalmente en la cultura enajenada que define Bonfil como: "este ámbito se forma con los elementos culturales que son propios del grupo, pero sobre los cuales ha perdido la capacidad de decidir: es decir, son elementos que forman parte del patrimonio cultural del grupo pero que se ponen en juego a partir de decisiones ajenas" (Bonfil 1988). Frente a esto el más claro caso es la práctica del turismo, ya que en este se han puesto muchas actividades tradicionales para obtener ganancias económicas; ejemplos de ello son la pesca tradicional que tiene un valor de 60 mil pesos, relatar cuentos tradicionales 100 mil pesos, la venta de elementos artesanales, avistamiento de delfines o incluso caminar entre la selva ahora tienen un valor económico y se hacen a petición de los turistas.

\section{Conclusiones}

La globalización es un fenómeno que ha venido desarrollándose en el mundo desde hace un buen tiempo, los autores que abordan el tema, difieren frente al tiempo que este proceso lleva desarrollándose, lo cierto es que ha llegado a todos los rincones del mundo, inicialmente afectando las estructuras de los Estados por medio del control económico, y que gradualmente fue incrustándose en todos los aspectos de la vida de las Naciones.

La masificación de las comunicaciones conlleva a la occidentalización de los pueblos, estandarizando las costumbres, gustos y necesidades, generando la pérdida de los valores, costumbres y tradiciones propios de las naciones. La comunidad indígena no ha escapado a este proceso, esta investigación permitió evidenciar cómo los valores, costumbres y necesidades de occidente se ven en una comunidad que se encuentra en la mitad de la selva amazónica, donde la necesidad de dinero está en el diario vivir, y la preocupación por un empleo o del rebusque está presente para todos en la comunidad, porque lo que produce la tierra ya no alcanza para cubrir lo que el dinero si puede. Por otro lado, se reconoce que el proceso de globalización han traído a las comunidades indígenas muchas tecnologías y procesos que les han permitido tener una visión más grande del mundo, de simplificar muchas de sus prácticas, no es lo mismo talar madera con hacha que con motosierra, ni estudiar con luz de vela a hacerlo con una estructura solar y con computadores, lo importante dentro del proceso de

${ }^{12}$ Cabe hacer claridad que no se condena ninguna de estas practicas, ya que muchos elementos han facilitado su estilo de vida, sin embargo si da a lugar a reflexionar frente cuales son las necesidadaes reales para vivir bien y cuales otras son producto de la occidentalizacion del amazonas. 
globalización es buscar los puntos de equilibrio, saber tomar lo bueno que trae y hacer resistencia a las cosas negativas, y esto es algo que la comunidad ha venido desarrollando. En este sentido emerge un reto para las diversas disciplinas que permita un dialogo interdisciplinar para crear junto con la comunidad estrategias reales logrando encontrar estos puntos de equilibrio, partiendo de un diagnóstico participativo, que no debe ir enfocado a la idea clásica de identificar y jerarquizar problemáticas que tiene la comunidad, sino más bien de reconocer las fortalezas y las propuestas de acción que las personas plantean desde su experiencia, identidad y cultura como indígenas, que en últimas es lo que puede garantizar que el conocimiento de las disciplinas se pongan al servicio de la comunidad, se reconozca y respete la particularidad.

Finalmente, el proceso desarrollado permite comprender la postura de la comunidad de Mocagua; esto gracias a su participación activa en el tiempo de inmersión que el equipo de investigadores realizó y en el cual presenció de primera mano, la vida diaria de las personas, sus alegrías y tristezas, las estrategias que usan cada día para sobrevivir y los significados que otorgan a sus tradiciones, y a la modernidad.

La comunidad hoy en día reconoce estar en una transición, entre la conservación de lo tradicional, el fortalecimiento de su cultura y los nuevos retos que plantea el mundo actual. Frente a esto han decidido proteger sus recursos tangibles como la fauna, flora, el agua y la tierra, enseñar a los niños el valor del territorio y fortalecer en ellos su patrimonio cultural Tikuna, organizarse como comunidad para decidir sobre las opciones que más beneficios pueden traer sin afectar la naturaleza, para alcanzar sus objetivos y proyecciones que se piensan en el futuro.

El Trabajo Social es una profesión bien reconocida en el abordaje de la mayoría de las problemáticas occidentales que ahora son propias de la comunidad indígena, a lo largo de su historia se ha planteado la necesidad de investigar e intervenir en realidades problemáticas, buscando del reconocimiento de la particularidad de los casos y de proponer estrategias y planes de mejora. Para la comunidad el abordaje puede realizarse desde el Trabajo social, En el ámbito familiar es clave el reconocimiento de los patrones culturales propios de los Tikuna, pero también de las transformaciones internas de la estructura familiar que han implantado años de educación religiosa, en este sentido es valioso reconocer cuales son las relaciones de poder conflictivas dentro de la familia Tikuna, y hasta qué punto pueden ser transformadas sin vulnerar su construcción cultural, por otro lado es necesario analizar cómo se construyen los vínculos al interior de la familia indígena, si bien el desarrollo del trabajo de campo identificó que la mayoría de hogares son de tipo nuclear con jefatura masculina, surge la inquietud frente a cómo se comporta el ciclo familiar, si existen otras tipologías de familia, y como se conforman desde la cultura propia de la comunidad o son producto del proceso de globalización.

Desde el trabajo social se pude concluir que el desarrollo de este trabajo aporta a la fundamentación disciplinar desde la interculturalidad, ya que como investigadores y trabajadores sociales no solo se tiene la oportunidad de comprender un contexto sino también de trabajar con la comunidad, se identificaron las transformaciones que atraviesa una comunidad específica, pero que al ser contrastadas con estudios de otras comunidades, muestran que los impactos de la globalización no son fenómenos aislados, son una realidad que enfrentan las comunidades indígenas en el país. Estos impactos se traducen en problemas sociales que afectan a las personas y sus familias, en trasformaciones económicas y políticas internas y de sus proyectos de vida.

De esta forma se puede proponer como ejes de acción para los profesionales en ciencias sociales que busquen adentrar su actuar en el ámbito de medio ambiente, aquellos que estén orientados a decodificar las relaciones estrechas, que entabla la población con la naturaleza, la forma como su construcción cultural la asimila y trata de transformarla y los sentidos y significados que para ellos representa, lo que en realidad devela su intención de conservación, transformación o destrucción. Este tipo de actuar debe ser "consecuente con la biodiversidad que defienden y la no-violencia sobre el ambiente natural, asumiendo la diversidad étnica y cultural desde una actitud comprensiva y dialógica".

\section{Referencias bibliográficas}

ACNUR. Agencia de la ONU para los refugiados. (1992). ISES\%20INDEPE (último acceso: 15 de Enero de 2016). Amador, M. (1998). Redes telemáticas y educación. 
Amat, B., Ghiotto, L. y Percovich. (2002)La Globalización Neoliberal y las nuevas redes de resistencia global. Buenos Aires: Centro Cultural de la Cooperación.

Amitai, E. (2007). La dimensión moral: Hacia una nueva economía. Ediciones Palabra.

Anderson, B. (1993). «Comunidades Imaginarias. reflexiones sobre el origen y la difusión del nacionalismo.» En Comunidades Imaginadas, de Benedict A., 157. Mexico D, F: Verso, Londres y Nueva York.

Arango, E. R. y Sánchez. (2004). Ministerio del Interior. h t t p s: // w w w. minin t e rior.gov. co/sit e s / default/files/upload/SIIC/PueblosIndigenas/pueblo_tikun a.pdf

Bengoa, J. (2000). La emergencia indígena en América Latina. Santiago de Chile: Fondo de Cultura Económica.

Bisquerra, R. (2004). Metodología de la investigación educativa. Madrid: La Muralla.

Blanco, H., Togeiro, L. y Gakkagher K. (2005). Globalización y Medio Ambiente. Lecciones desde las Américas. Santiago de Chile: RIDES-GDAE,.

Bonfil, G. (1988). Teoría del control cultural en los procesos étnicos. Brasilia: Universidad de Brasilia.

Cancillería de Colombia. Cancillería Colombia. (2002). http://www.cancilleria.gov.co/international/regional/amaz on.

Cárcamo, S. (s.f.). Universidad Nacional Experimental Simón Rodríguez. ht t p://www. postgrado.unesr. edu.ve/acontece/es/todosnumeros/num16/01_03/Solang e_Carcamo.pdf

Castro, P. et al. (1996). «Teoría de las practicas sociales.» Complutum 6 (2) pp: 35-48.

Chomsky, N. (2004). La Globalización tiene poco de libre mercado. http://galeon.hispavista.com/bvchomsky/ textos/glob02.html

Cohen. 2000: 46.

Congreso de La República de Colombia. (1991). Constitución Política de Colombia. Bogotá.

Comisión Económica para América Latina y el Caribe y programa de las Naciones Unidas para el Medio ambiente. (2003).

Corporación para el Desarrollo Sostenible del Sur de la A m a zonia - Corpo a m a zonia. (s.f.). http:/www.corpoamazonia.gov.co/region/Amazonas/Cart ografia/Ama_normativo.html.

Corte Constitucional. Corte Constitucional. (s.f.). http://www.corteconstitucional.gov.co/RELATORIA/2012 /T-001-12.html.

Curaca, entrevista de David Guantiva y Sebastian Valencia. (21 de Diciembre de 2015).

Deruyttere, A. (2001). Pueblos indígenas, globalización y desarrollo con identidad: algunas reflexiones de estrategia. Banco interamericano de desarrollo.

Director, escuela, entrevista de David Guantiva y Sebastian Valencia. (8 de Enero de 2016).
Escobar, A. (2010). Territorios de diferencia. Lugar, movimientos, vida, redes. Popayán: Samava impresiones.

Fontaine, G. (2006). «La Globalización de la Amazonia. Una perspectiva Andina.» Revista de Ciencias Sociales (Facultad Latinoamericana de Ciencias Sociales), $\mathrm{n}^{0} 25$ pp: 6-8.

Fontaine, L. (2003). «El mambe frente al dinero entre los yucuna del Amazonas.» Revista Colombiana de Antropología. $\mathrm{n}^{\circ} 1$. http://www.icanh.gov.co/ index.php?idcategoria $=3995$

Galeano, E. (2005). «El imperio del Consumo.» Revista digital Rebelión, $\mathrm{n}^{\circ} 2$.

Gonzalez, U. L. (2000). «Reflexiones en torno al concepto de

Globalizacion.» Revista de Humanidades. Tecnológico de

Monterrey.

Goulard. 1998: 173-174.

Guantiva, D. y Valencia, S. (2015). «Guía de observación.» Mocagua, Amazonas, 24 de Diciembre.

Guantiva, D. y Valencia, S. (2016). Mocagua, Director escuela, entrevista de David Guantiva y Valencia Sebastián. Amazonas, Mocagua.

Guantiva, D. y Valencia, S. (2015). Mujer, Mocagua, entrevista de David Guantiva y Sebastian Valencia. Amazonas, Mocagua.

Guba, E, y M Lincoln. Paradigmas en competencia en la investigación cualitativa. Sonora, 2002.

Gudynas, E. (2005). «La Nueva Geografía Amazónica: entre la globalización y el regionalismo. OD.» La Paz.

Guantiva, D. y Valencia, S. (2016). Habitante, entrevista de David Guantiva y Valencia Sebastián.

Ianni, O. (2006). Teorías de la Globalización. Mexico: siglo XXI editores.

Instituto Socioambiental ISA. «Povos Indigenas no Brasil.» ISA. (2008). http://pib.socioambiental.org/es/povo/ ticuna/1344

Leon, H. (s.f). «Tomando masato en Mocagua (mocawa).» En Tomando masato en Mocagua (mocawa), de Hector Leon, 15-25. Mocagua.

LIDEMA. (2011). «Liga para la defensa del Medio Ambiente.» bivica. http://www.bivica.org/upload/medios-vida.pdf

Lopez G. y Leonor, C. (2002). «Los Ticuna frente a los procesos de nacionalización en la frontera entre Brasil, Colombia y Perú.» Revista Colombiana de Antropología, 38, 77-104. http://www. scielo.org.co/scielo.php? script $=$ sci_arttext\&pid $=$ S0486-65252002000100004 $\& \operatorname{lng}=\mathrm{en} \& \mathrm{t} \operatorname{lng}=\mathrm{es}$.

Marin U, G. (1988). Leticia: su historia. issuu.com/web-unaamazonia/docs/3notifeb12

Martinez, J. (1999). Globalización: Elementos para el debate. Editado por Miguel Angel Porrua. http://res.uniandes.edu.co/view.php/171/view.php

Martinez, M. (2000). La investigación cualitativa etnográfica en educación. Bogotá: Círculo de la lectura alternativa.

Mead, G. (1974). Self and Society. Chicago: University of 
Chicago Press.

Mesino, L. (2009). «La globalización económica y sus implicaciones socio -culturales en América Latina.» Revista de Ciencias Sociales Scielo, Vol. 15, nº 1 pp 126138.

Ministerio del Interior. (2004). Pueblos indígenas / pueblo Ticuna. https://www.mininterior.gov.co/ sites/default/files/upload/SIIC/PueblosIndigenas/pueblo_t ikuna.pdf

Museu, G. (2005). Ticunas Brasileros, Colombianos y Peruanos: etnicidad y nacionalidad en la región de fronteras del alto amazonas/Solimoes. www.tesis.bioetica.org/tic 2.html

Ochoa, G, I. (2007). «El turismo ¿una nueva bonanza de la amazonia?» Editado por Universidad Nacional. Fronteras en la Globalización, Localidad, Biodiversidad y libre comercio en la Amazonia.

OIT. Organización internacional del Trabajo. 7 de Junio de 1989. http://www.corteconstitucional.gov.co/rela toria/CONVENIO\%20169\%20DE\%20LA\%20OIT.\%20SO BRE\%20PUEBLOS\%20INDIGENAS\%20Y\%20TRIBALE S\%20EN\%20PAISES\%20INDEPENDIENTES.php (último acceso: 20 de Marzo de 2016).

OIT. «Seminario sobre Recopilación y desglose de datos relativos a los pueblos indígenas.» Enero 2004: 9

Ortiz, A. Enfoques y métodos de investigación en las ciencias sociales. Bogotá: Ediciones de la U, 2015.

Palacio, G. "Amazonia desde adentro, Aportes a la investigación de la amazonia colombiana.» Universidad Nacional de Colombia sede Amazonia, s.f.: 17-18.

Panduro, C, entrevista de David Guantiva y Sebastian Valencia. Funcionario de Parque Nacional Natural Amacayacu Amazonas, Mocagua, (6 de Enero de 2016).

Parques Nacionales Naturales de Colombia. Parques Nacionales Naturales de Colombia. 2009. http://www.parquesnacionales.gov.co/portal/es/organizaci on/ (último acceso: 01 de 2016).

Pinzon Forero, A. La colonización militar y el conflicto colomboperuano. Editado por ACORE. Vers. Vol 1. 1960. http://tesis.bioetica.org/tic2.htm.

Quintana, A.R (2017), Medicina tradicional en la comunidad de San Basilo de PalenqueÕ'dRevista Nova.Vol. 13 (25) 67-93

Rodríguez, y Valdeoriola. Metodología de la investigación. Universidad Oberta de Cataluña, s.f.

Soto, M. Gómez. «Viviendo en efectivo: la economía de los Tikuna de Macedonia.» Ebrary. Editado por Universidad de los Andes. 2010. http://www.ebrary.com (último acceso: 17 de Septiembre de 2015).

Torres, J. Zapata. «Espacio y Territorio Sagrado, lógica del ordenamiento territorial indígena.» Editado por Universidad Nacional de Colombia. 20 de marzo de 2010. h t t p ://www.alberdi.de/E S PA C I O \% $20 \% 20 \mathrm{Y} \%$ 20TERRITORIO\%20SAGRADO-Jair,actu,02.06.07.pdf (último acceso: 17 de Septiembre de 2015).

Torres, W., y Fajardo, G. «Introducción a la Colombia Amerindia. Ticuna.» Editado por Editoria presencia. 1987. h t t p : / / w w w. b a n r e p c u l t u r a l. o r g / blaavirtual/antropologia/amerindi/ticuna.htm (último acceso: 07 de 2015).

Universidad del Rosario. Jurisprudencia. 1991. http://www.urosario.edu.co/jurisprudencia/catedra-vivaintercultural/ur/Legislacion-Internacional/ (último acceso: 23 de Octubre de 2015).

-Universidad del Rosario. s.f. http://www.urosario. edu.co/jurisprudencia/catedra-viva-inter cultural/ ur/LaConsulta-Previa/Que-es-la-Consulta-Previa/ (último acceso: 10 de Marzo de 2016).

Universidad Nacional Abierta y a Distancia. Lección 6. Investigación Exploratoria Descriptiva Correlacional y Explicativa. 2006. Http://datateca.unad.edu.co/ contenidos/100104

Zamudio, H. (2005). «Amazonia. Entre la Integración Regional y la Globalización.» Luna Azul, nº 20 pp: 2-7. 